\title{
Master integrals for massless three-loop form factors
}

\author{
Tobias Huber*a,b \\ ${ }^{a}$ Institut für Theoretische Physik E, RWTH Aachen University, \\ D-52056 Aachen, Germany \\ ${ }^{b}$ Fachbereich 7, Universität Siegen, \\ Walter-Flex-Str. 3, D-57068 Siegen, Germany \\ E-mail: huberetp1.physik.uni-siegen.de
}

\begin{abstract}
We summarize the results for the master integrals of the three-loop quark and gluon form factor in massless QCD. Working in dimensional regularization we extract poles up to $1 / \varepsilon^{6}$. The computational techniques involve, among others, the expansion of higher transcendental functions and the Mellin-Barnes method. The coefficients of the Laurent expansion in $\varepsilon$ are given either analytically or numerically to high precision.
\end{abstract}

RADCOR 2009 - 9th International Symposium on Radiative Corrections (Applications of Quantum Field Theory to Phenomenology)

October 25-30 2009

Ascona, Switzerland

* Speaker. 


\section{Introduction and computational methods}

The quark form factor $\gamma^{*} \rightarrow q \bar{q}$ and gluon form factor $H \rightarrow g g$ (effective coupling) are the simplest processes containing IR divergences at higher orders in massless QFT, and therefore are of particular interest in many aspects. They have been used to predict the IR pole structure of multi-leg amplitudes [1-4]. The form factors can also be exploited to extract resummation coefficients [5,6], and they enter the purely virtual corrections to a number of collider reactions (Drell-Yan process, Higgs production and decay, DIS). Besides phenomenological applications, a major motivation for obtaining analytic results at three-loop order and beyond is finding and understanding structures in massless gauge theories that generalize to an arbitrary number of loops. Much progress has been achieved in the prediction of all-order singularity structures in QCD [7-9], in conjectures about the all-orders behaviour of maximally supersymmetric Yang-Mills theories (see e.g. [10-12]) and in investigations of the finiteness of $\mathrm{N}=8$ supergravity, see e.g. [13-15].

The two-loop corrections to the massless-quark [16-18] and gluon $[19,20]$ form factors were computed in dimensional regularisation with $D=4-2 \varepsilon$ to order $\varepsilon^{0}$ and subsequently extended to all orders in $\varepsilon$ in ref. [21]. The three-loop form factors to order $\varepsilon^{-1}$ (and $\varepsilon^{0}$ for contributions involving fermion loops in the quark form factor) were computed in [6,22]. Recently, also the three-loop form factors through $\varepsilon^{0}$ became available [23] (see also [24]).

In order to calculate the quark and gluon form factors at higher orders in perturbation theory, the amplitudes are reduced to a small set of master integrals by means of algebraic reduction procedures [25-31]. At the three-loop level, the reduction results in 22 master integrals. Eight of them are products of one-loop and two-loop vertex functions or three-loop two-point functions, both of which are known to sufficiently high orders in $\varepsilon[21,25,26,32,33]$. The remaining fourteen masters are genuine three-loop vertex functions which are depicted in Fig. 1. Each topology contains only one master integral, and corresponds to two-particle cuts of the master integrals for massless fourloop off-shell propagator integrals [34]. Working in dimensional regularisation with $D=4-2 \varepsilon$ and expanding the master integrals in a Laurent series in $\varepsilon$, the finite part of the three-loop form factors requires the extraction of all coefficients through (polylogarithmic) weight six, i.e. coefficients containing terms up to $\pi^{6}$ or $\zeta_{3}^{2}$.

The computational methods that we use during the calculation are manifold. The easier integrals $\left(A_{5,1}, A_{5,2}, A_{6,1}\right)$ contain only $\Gamma$-functions and their expansion is straightforward. The more complicated masters $A_{6,3}, A_{7,1}, A_{7,2}$, and $A_{7,4}$ are also represented in a closed form in terms of hypergeometric functions of unit argument. The latter are expanded in $\varepsilon$ by means of HypExp [35, 36]. The remaining integrals possess multiple Mellin-Barnes (MB) [37-41] representations. Their analytic continuation to $\varepsilon=0$ was done with MB [42] and MBresolve [43]. These packages were also used for numerical cross checks. In addition we performed numerical checks with the sector decomposition methods of $[44,45]$ and the FIESTA [46] package.

As analytic techniques we apply Barnes's lemmas and the theorem of residues to the multiple MB integrals, and insert integral representations of higher transcendental functions where appropriate. We also make use of the HPL [47] and barnesroutines [48] packages, as well as the nested sums algorithm $[49,50]$. 


\section{Results}

Below, we list the results [51-53] for the fourteen genuine vertex-type master integrals from Fig. 1. We use the following definitions,

$$
q^{2}=\left(p_{1}+p_{2}\right)^{2}, \quad \int[d k] \equiv \int \frac{d^{D} k}{(2 \pi)^{D}}, \quad S_{\Gamma}=\frac{1}{(4 \pi)^{D / 2} \Gamma(1-\varepsilon)},
$$

and moreover we tacitly assume that all propagators contain an infinitesimal $+i \eta$ with $\eta>0$.

\subsection{Integrals with five or six propagators}

We start with three integrals that can be displayed in a closed form in terms of $\Gamma$-functions only. Their expansion about $\varepsilon=0$ can be easily performed with standard computer algebra programs.

$$
\begin{aligned}
A_{5,1} & =\int[d k] \int[d l] \int[d r] \frac{1}{\left(k+p_{1}\right)^{2}\left(k-p_{2}\right)^{2} l^{2}(k+l+r)^{2} r^{2}} \\
& =i S_{\Gamma}^{3}\left[-q^{2}-i \eta\right]^{1-3 \varepsilon} \frac{\Gamma^{6}(1-\varepsilon) \Gamma(2 \varepsilon) \Gamma(3 \varepsilon) \Gamma(1-3 \varepsilon)}{(1-2 \varepsilon)(2-3 \varepsilon) \Gamma(3-4 \varepsilon)} \\
A_{5,2} & =-i S_{\Gamma}^{3}\left[-q^{2}-i \eta\right]^{1-3 \varepsilon} \frac{\Gamma^{7}(1-\varepsilon) \Gamma(\varepsilon) \Gamma(3 \varepsilon) \Gamma(1-3 \varepsilon)}{(1-2 \varepsilon) \Gamma(2-2 \varepsilon) \Gamma(3-4 \varepsilon)} \\
A_{6,1} & =-i S_{\Gamma}^{3}\left[-q^{2}-i \eta\right]^{-3 \varepsilon} \frac{\Gamma^{7}(1-\varepsilon) \Gamma^{2}(\varepsilon) \Gamma(3 \varepsilon) \Gamma^{2}(1-3 \varepsilon)}{\Gamma^{2}(2-2 \varepsilon) \Gamma(2-4 \varepsilon)} .
\end{aligned}
$$

The next integral, $A_{6,2}$, can be written in terms of a two-fold Mellin-Barnes representation.

$$
\begin{aligned}
A_{6,2}= & -i S_{\Gamma}^{3}\left[-q^{2}-i \eta\right]^{-3 \varepsilon} \frac{\Gamma^{3}(1-\varepsilon) \Gamma(3 \varepsilon) \Gamma^{2}(1-3 \varepsilon)}{\Gamma(1-2 \varepsilon) \Gamma(2-4 \varepsilon)} \int_{c_{1}-i \infty}^{c_{1}+i \infty} \frac{d w_{1}}{2 \pi i} \int_{c_{2}-i \infty}^{c_{2}+i \infty} \frac{d w_{2}}{2 \pi i} \\
& \times \frac{\Gamma\left(-1+3 \varepsilon-w_{1}\right) \Gamma\left(-1+2 \varepsilon-w_{1}\right) \Gamma\left(2-4 \varepsilon+w_{1}\right) \Gamma\left(-w_{2}\right) \Gamma\left(w_{2}-w_{1}\right)}{\Gamma\left(3 \varepsilon-w_{1}\right) \Gamma\left(2-4 \varepsilon+w_{2}\right) \Gamma\left(2-4 \varepsilon+w_{1}-w_{2}\right)} \\
& \times \Gamma\left(1-\varepsilon+w_{2}\right) \Gamma\left(1-\varepsilon+w_{1}-w_{2}\right) \Gamma\left(1-2 \varepsilon+w_{2}\right) \Gamma\left(1-2 \varepsilon+w_{1}-w_{2}\right) .
\end{aligned}
$$

The contour integrals in the complex plane can be chosen as straight lines parallel to the imaginary axis, i.e. the real parts $c_{1}$ and $c_{2}$ along the curves are constant. Their values, together with that of $\varepsilon$, must be chosen such as to separate left poles of $\Gamma$-functions from right ones [38-40], which is achieved by taking $c_{1}=-6 / 5, c_{2}=-1 / 2$, and $-1 / 15<\varepsilon<3 / 20$. The analytic continuation to $\varepsilon=0$ is therefore trivial, and after summing the residues one obtains

$$
\begin{aligned}
A_{6,2}= & i S_{\Gamma}^{3}\left[-q^{2}-i \eta\right]^{-3 \varepsilon}\left[-\frac{2 \zeta_{3}}{\varepsilon}-18 \zeta_{3}-\frac{7 \pi^{4}}{180}+\left(-122 \zeta_{3}-\frac{7 \pi^{4}}{20}+\frac{2 \pi^{2}}{3} \zeta_{3}-10 \zeta_{5}\right) \varepsilon\right. \\
& \left.+\left(-738 \zeta_{3}-\frac{427 \pi^{4}}{180}+6 \pi^{2} \zeta_{3}-90 \zeta_{5}+\frac{163 \pi^{6}}{7560}+76 \zeta_{3}^{2}\right) \varepsilon^{2}+\mathscr{O}\left(\varepsilon^{3}\right)\right] .
\end{aligned}
$$




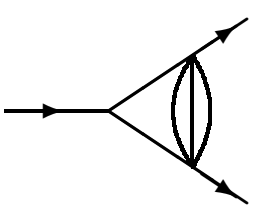

$A_{5,1}$

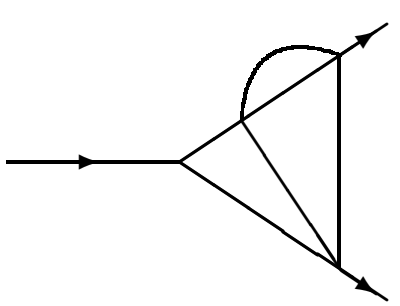

$A_{6,3}$

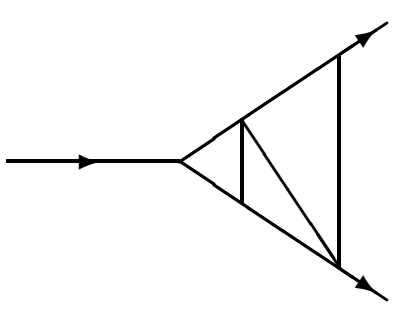

$A_{7,3}$

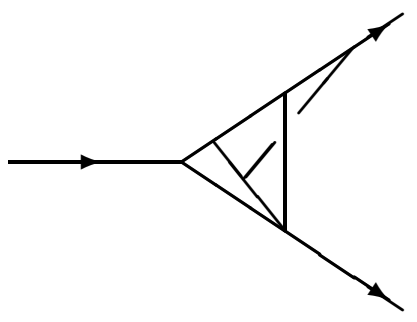

$A_{8}$

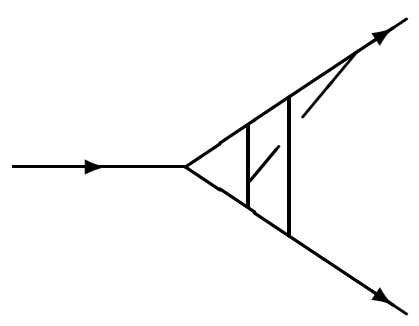

$A_{9,2}$
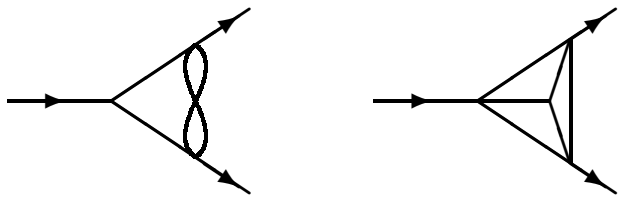

$A_{6,1}$

$A_{6,2}$

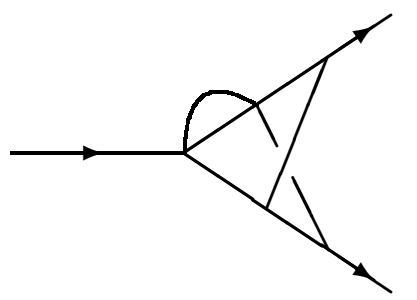

$A_{7,1}$

$A_{7,2}$

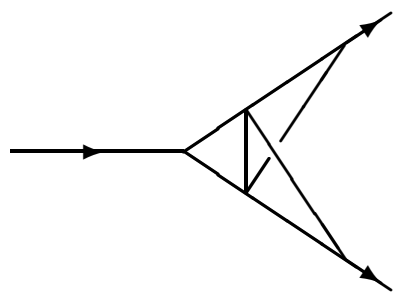

$A_{7,4}$

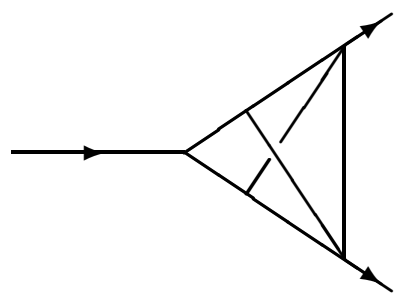

$A_{7,5}$

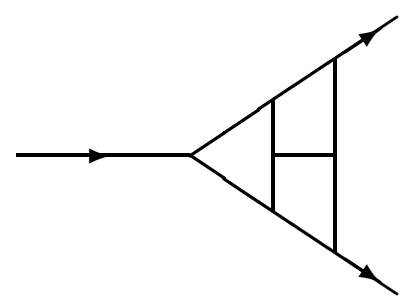

$A_{9,1}$

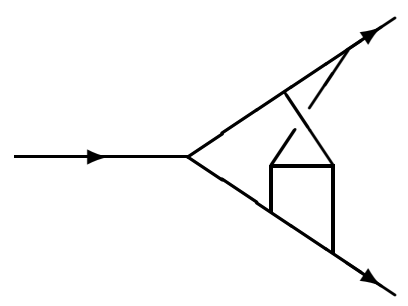

$A_{9,4}$

Figure 1: The fourteen genuine vertex-type master integrals. The incoming momentum is $q=p_{1}+p_{2}$. Outgoing momenta are taken to be on-shell and massless, $p_{1}^{2}=p_{2}^{2}=0$. 
In Ref. [36], two more orders of the $\varepsilon$-expansion can be found. The next integral, $A_{6,3}$, can be displayed in a closed form valid to all orders in $\varepsilon$ in terms of hypergeometric functions of unit argument. The latter are expanded in $\varepsilon$ by means of the Mathemat ica package HypExp $[35,36]$,

$$
\begin{aligned}
A_{6,3}= & -i S_{\Gamma}^{3}\left[-q^{2}-i \eta\right]^{-3 \varepsilon} \frac{\Gamma^{7}(1-\varepsilon) \Gamma(-1+3 \varepsilon)}{(1-2 \varepsilon)^{2}(1-3 \varepsilon) \Gamma(2-4 \varepsilon)} \\
& \times\left[-\frac{\Gamma(2-3 \varepsilon) \Gamma(2 \varepsilon) \Gamma(\varepsilon) \Gamma(2-2 \varepsilon)}{\Gamma^{2}(1-\varepsilon)}+{ }_{3} F_{2}(1,1-\varepsilon, 1-2 \varepsilon ; 2-2 \varepsilon, 2-3 \varepsilon ; 1)\right] \\
= & i S_{\Gamma}^{3}\left[-q^{2}-i \eta\right]^{-3 \varepsilon}\left[-\frac{1}{6 \varepsilon^{3}}-\frac{3}{2 \varepsilon^{2}}-\left(\frac{55}{6}+\frac{\pi^{2}}{6}\right) \frac{1}{\varepsilon}-\frac{95}{2}-\frac{3 \pi^{2}}{2}+\frac{17 \zeta_{3}}{3}+\left(-\frac{1351}{6}\right.\right. \\
& \left.-\frac{55 \pi^{2}}{6}-\frac{\pi^{4}}{90}+51 \zeta_{3}\right) \varepsilon+\left(-\frac{2023}{2}-\frac{95 \pi^{2}}{2}-\frac{\pi^{4}}{10}+\frac{935 \zeta_{3}}{3}+\frac{10 \pi^{2} \zeta_{3}}{3}+65 \zeta_{5}\right) \varepsilon^{2} \\
& \left.+\left(\frac{7 \pi^{6}}{54}-\frac{26335}{6}-\frac{1351 \pi^{2}}{6}-\frac{11 \pi^{4}}{18}+1615 \zeta_{3}+30 \pi^{2} \zeta_{3}-\frac{268 \zeta_{3}^{2}}{3}+585 \zeta_{5}\right) \varepsilon^{3}+\mathscr{O}\left(\varepsilon^{4}\right)\right] .
\end{aligned}
$$

\subsection{Integrals with seven or eight propagators}

Since the explicit expressions for the integrals that follow are very lengthy, we summarize in this paragraph the respective techniques and subsequently only give the results of the Laurent expansion in $\varepsilon$. Integrals $A_{7,1}, A_{7,2}$, and $A_{7,4}$ reveal a closed form in terms of hypergeometric functions of unit argument, whose $\varepsilon$ expansions are carried out with HypExp [35, 36]. Integrals $A_{7,3}, A_{7,5}$, and $A_{8}$ possess multiple Mellin-Barnes representations which are three-, four- and fourfold respectively. Only in the case of $A_{8}$ the analytic continuation to $\varepsilon=0$ is non-trivial and is carried out with MB [42]. The explicit results read

$$
\begin{aligned}
A_{7,1}= & i S_{\Gamma}^{3}\left[-q^{2}-i \eta\right]^{-1-3 \varepsilon}\left[\frac{1}{4 \varepsilon^{5}}+\frac{1}{2 \varepsilon^{4}}+\left(1-\frac{\pi^{2}}{6}\right) \frac{1}{\varepsilon^{3}}+\left(2-\frac{\pi^{2}}{3}-10 \zeta_{3}\right) \frac{1}{\varepsilon^{2}}\right. \\
+ & \left(4-\frac{2 \pi^{2}}{3}-\frac{11 \pi^{4}}{45}-20 \zeta_{3}\right) \frac{1}{\varepsilon}+\left(8-\frac{4 \pi^{2}}{3}-\frac{22 \pi^{4}}{45}-40 \zeta_{3}+\frac{14 \pi^{2} \zeta_{3}}{3}-88 \zeta_{5}\right) \\
+ & \left.\left(16-\frac{8 \pi^{2}}{3}-\frac{44 \pi^{4}}{45}-\frac{943 \pi^{6}}{7560}-80 \zeta_{3}+\frac{28 \pi^{2} \zeta_{3}}{3}+196 \zeta_{3}^{2}-176 \zeta_{5}\right) \varepsilon+\mathscr{O}\left(\varepsilon^{2}\right)\right] \\
A_{7,2}= & i S_{\Gamma}^{3}\left[-q^{2}-i \eta\right]^{-1-3 \varepsilon}\left[\frac{\pi^{2}}{12 \varepsilon^{3}}+\left(\frac{\pi^{2}}{6}+2 \zeta_{3}\right) \frac{1}{\varepsilon^{2}}+\left(\frac{\pi^{2}}{3}+\frac{83 \pi^{4}}{720}+4 \zeta_{3}\right) \frac{1}{\varepsilon}\right. \\
& +\left(\frac{2 \pi^{2}}{3}+\frac{83 \pi^{4}}{360}+8 \zeta_{3}-\frac{5 \pi^{2} \zeta_{3}}{3}+15 \zeta_{5}\right) \\
& \left.+\left(\frac{4 \pi^{2}}{3}+\frac{83 \pi^{4}}{180}+\frac{2741 \pi^{6}}{90720}+16 \zeta_{3}-\frac{10 \pi^{2} \zeta_{3}}{3}-73 \zeta_{3}^{2}+30 \zeta_{5}\right) \varepsilon+\mathscr{O}\left(\varepsilon^{2}\right)\right]
\end{aligned}
$$




$$
\begin{aligned}
A_{7,3}= & i S_{\Gamma}^{3}\left[-q^{2}-i \eta\right]^{-1-3 \varepsilon}\left[\left(-\frac{\pi^{2} \zeta_{3}}{6}-10 \zeta_{5}\right) \frac{1}{\varepsilon}-\frac{119 \pi^{6}}{2160}-\frac{31}{2} \zeta_{3}^{2}+\mathscr{O}(\varepsilon)\right], \\
A_{7,4}= & i S_{\Gamma}^{3}\left[-q^{2}-i \eta\right]^{-1-3 \varepsilon}\left[\frac{6 \zeta_{3}}{\varepsilon^{2}}+\left(\frac{11 \pi^{4}}{90}+36 \zeta_{3}\right) \frac{1}{\varepsilon}+\left(\frac{11 \pi^{4}}{15}+216 \zeta_{3}-2 \pi^{2} \zeta_{3}+46 \zeta_{5}\right)\right. \\
\left.+\left(\frac{22 \pi^{4}}{5}-\frac{19 \pi^{6}}{270}+1296 \zeta_{3}-12 \pi^{2} \zeta_{3}-282 \zeta_{3}^{2}+276 \zeta_{5}\right) \varepsilon+\mathscr{O}\left(\varepsilon^{2}\right)\right], & \\
A_{7,5}= & i S_{\Gamma}^{3}\left[-q^{2}-i \eta\right]^{-1-3 \varepsilon}\left[2 \pi^{2} \zeta_{3}+10 \zeta_{5}+\left(12 \pi^{2} \zeta_{3}+60 \zeta_{5}+\frac{11 \pi^{6}}{162}+18 \zeta_{3}^{2}\right) \varepsilon+\mathscr{O}\left(\varepsilon^{2}\right)\right] . \\
A_{8}= & i S_{\Gamma}^{3}\left[-q^{2}-i \eta\right]^{-2-3 \varepsilon}\left[\frac{8 \zeta_{3}}{3 \varepsilon^{2}}+\left(\frac{5 \pi^{4}}{27}-8 \zeta_{3}\right) \frac{1}{\varepsilon}+24 \zeta_{3}-\frac{5 \pi^{4}}{9}-\frac{52}{9} \pi^{2} \zeta_{3}+\frac{352}{3} \zeta_{5}\right. \\
+ & \left.\left(-72 \zeta_{3}+\frac{5 \pi^{4}}{3}+\frac{52}{3} \pi^{2} \zeta_{3}-352 \zeta_{5}+\frac{1709 \pi^{6}}{8505}-\frac{332}{3} \zeta_{3}^{2}\right) \varepsilon+\mathscr{O}\left(\varepsilon^{2}\right)\right] .
\end{aligned}
$$

\subsection{Integrals with nine propagators}

Integrals $A_{9,1}, A_{9,2}$, and $A_{9,4}$ possess a six-fold MB representation each. The analytic continuation, carried out with MB [42], involves in each case approximately 200 steps. We apply the techniques described in section 1 , and stress that all results were obtained by purely analytic steps. We have

$$
\begin{aligned}
A_{9,1}= & \int[d k] \int[d l] \int[d r] \frac{1}{k^{2}\left(k+p_{1}\right)^{2}(k+l)^{2}(k-r)^{2}(l+r)^{2}\left(l+p_{2}\right)^{2} l^{2}\left(r+p_{1}\right)^{2}\left(r-p_{2}\right)^{2}} \\
= & i S_{\Gamma}^{3}\left[-q^{2}-i \eta\right]^{-3-3 \varepsilon}\left[-\frac{1}{18 \varepsilon^{5}}+\frac{1}{2 \varepsilon^{4}}+\left(-\frac{53}{18}-\frac{4 \pi^{2}}{27}\right) \frac{1}{\varepsilon^{3}}+\left(\frac{29}{2}+\frac{22 \pi^{2}}{27}-2 \zeta_{3}\right) \frac{1}{\varepsilon^{2}}\right. \\
& +\left(\frac{158}{9} \zeta_{3}-\frac{129}{2}-\frac{8 \pi^{2}}{3}-\frac{20 \pi^{4}}{81}\right) \frac{1}{\varepsilon}+\left(\frac{537}{2}+6 \pi^{2}-\frac{578}{9} \zeta_{3}+\frac{322 \pi^{4}}{405}-\frac{14}{3} \pi^{2} \zeta_{3}-\frac{238}{3} \zeta_{5}\right) \\
& \left.+\left(-\frac{2133}{2}-4 \pi^{2}+158 \zeta_{3}-\frac{302 \pi^{4}}{135}-\frac{26}{3} \pi^{2} \zeta_{3}+\frac{826}{3} \zeta_{5}-\frac{2398 \pi^{6}}{5103}-\frac{466}{3} \zeta_{3}^{2}\right) \varepsilon+\mathscr{O}\left(\varepsilon^{2}\right)\right],
\end{aligned}
$$

$$
\begin{aligned}
A_{9,2}= & \int[d k] \int[d l] \int[d r] \frac{1}{k^{2}\left(k+p_{1}\right)^{2}\left(k-l+p_{1}\right)^{2}(k-r-l)^{2}(l+r)^{2}\left(l+p_{2}\right)^{2} l^{2}\left(r+p_{1}\right)^{2}\left(r-p_{2}\right)^{2}} \\
= & i S_{\Gamma}^{3}\left[-q^{2}-i \eta\right]^{-3-3 \varepsilon}\left[\frac{2}{9 \varepsilon^{6}}+\frac{5}{6 \varepsilon^{5}}+\left(-\frac{20}{9}-\frac{7 \pi^{2}}{27}\right) \frac{1}{\varepsilon^{4}}+\left(\frac{50}{9}-\frac{17 \pi^{2}}{27}-\frac{91}{9} \zeta_{3}\right) \frac{1}{\varepsilon^{3}}\right. \\
& +\left(-\frac{110}{9}+\frac{4 \pi^{2}}{3}-\frac{166}{9} \zeta_{3}-\frac{373 \pi^{4}}{1080}\right) \frac{1}{\varepsilon^{2}}+\left(\frac{170}{9}-\frac{16 \pi^{2}}{9}+\frac{494}{9} \zeta_{3}-\frac{187 \pi^{4}}{540}\right. \\
& \left.\left.+\frac{179}{27} \pi^{2} \zeta_{3}-167 \zeta_{5}\right) \frac{1}{\varepsilon}+(-670.0785 \pm 0.0326)+\mathscr{O}(\varepsilon)\right] . \\
A_{9,4}= & \int[d k] \int[d l] \int[d r] \frac{1}{k^{2}\left(k+p_{1}\right)^{2}(k-r)^{2}(k-r-l)^{2}(l+r)^{2}\left(l+p_{2}\right)^{2} l^{2}\left(r+p_{1}\right)^{2}\left(r-p_{2}\right)^{2}}
\end{aligned}
$$




$$
\begin{aligned}
= & i S_{\Gamma}^{3}\left[-q^{2}-i \eta\right]^{-3-3 \varepsilon}\left[\frac{1}{9 \varepsilon^{6}}+\frac{8}{9 \varepsilon^{5}}+\left(-1-\frac{10 \pi^{2}}{27}\right) \frac{1}{\varepsilon^{4}}+\left(-\frac{14}{9}-\frac{47 \pi^{2}}{27}-12 \zeta_{3}\right) \frac{1}{\varepsilon^{3}}\right. \\
& +\left(17+\frac{71 \pi^{2}}{27}-\frac{200}{3} \zeta_{3}-\frac{47 \pi^{4}}{810}\right) \frac{1}{\varepsilon^{2}}+(117.3999538 \pm 0.0000032) \frac{1}{\varepsilon} \\
& +(1948.167043 \pm 0.000025)+\mathscr{O}(\varepsilon)] .
\end{aligned}
$$

The numbers were obtained with MB . m [42]. We stress that all other terms in (2.14) - (2.16) were derived by purely analytic steps. The analytic result of the simple pole of $A_{9,4}$ can be extracted from [23]. It turns out that for each of the above integrals a corresponding one with an irreducible scalar product in the numerator can be chosen whose coefficients of the $\varepsilon$-expansion have homogeneous weight. The suitably chosen numerators are $r^{2}$ for $A_{9,1}$ and $A_{9,4}$, and $\left(l-p_{1}\right)^{2}$ for $A_{9,2}$. The property of homogeneous weight is very helpful when one uses the PSLQ algorithm [54], by means of which we obtain

$$
\begin{aligned}
A_{9,1}^{(n)}= & i S_{\Gamma}^{3}\left[-q^{2}-i \eta\right]^{-2-3 \varepsilon}\left[-\frac{1}{36 \varepsilon^{6}}-\frac{\pi^{2}}{18 \varepsilon^{4}}-\frac{14 \zeta_{3}}{9 \varepsilon^{3}}-\frac{47 \pi^{4}}{405 \varepsilon^{2}}\right. \\
& \left.+\left(-\frac{85}{27} \pi^{2} \zeta_{3}-20 \zeta_{5}\right) \frac{1}{\varepsilon}+\left(-\frac{1160 \pi^{6}}{5103}-\frac{137}{3} \zeta_{3}^{2}\right)+\mathscr{O}(\varepsilon)\right] \\
A_{9,2}^{(n)}= & i S_{\Gamma}^{3}\left[-q^{2}-i \eta\right]^{-2-3 \varepsilon}\left[-\frac{2}{9 \varepsilon^{6}}+\frac{7 \pi^{2}}{27 \varepsilon^{4}}+\frac{91 \zeta_{3}}{9 \varepsilon^{3}}+\frac{373 \pi^{4}}{1080 \varepsilon^{2}}\right. \\
& \left.+\left(-\frac{179}{27} \pi^{2} \zeta_{3}+167 \zeta_{5}\right) \frac{1}{\varepsilon}+(395.3405 \pm 0.0326)+\mathscr{O}(\varepsilon)\right] \\
A_{9,4}^{(n)}= & i S_{\Gamma}^{3}\left[-q^{2}-i \eta\right]^{-2-3 \varepsilon}\left[\frac{1}{9 \varepsilon^{6}}-\frac{10 \pi^{2}}{27 \varepsilon^{4}}-\frac{12 \zeta_{3}}{\varepsilon^{3}}-\frac{47 \pi^{4}}{810 \varepsilon^{2}}\right. \\
& \left.+(206.7612077 \pm 0.0000032) \frac{1}{\varepsilon}+(1237.300592 \pm 0.000035)+\mathscr{O}(\varepsilon)\right] .
\end{aligned}
$$

Since each topology contains only one master integral, each $A_{9, i}$ is related to its corresponding $A_{9, i}^{(n)}$. We established these relations with the Laporta algorithm $[27,28,30]$ and checked that they are filfilled by the above expressions.

The analytic expressions for the remaining coefficients are within reach. In the finite part of $A_{9,2}$ we are missing only $\sim 30$ terms, all four- and five-fold MB integrals. From what we can judge it is not possible to process these MB kernels by purely analytic steps. We therefore plan to break them down to lower-dimensional integrals over ordinary Feynman parameters and then use the PSLQ algorithm. In the case of $A_{9,4}$ we are left with $\mathscr{O}\left(10^{3}\right) \mathrm{MB}$ terms which are at most three-fold. We are confident that they can be processed by purely analytic steps.

\section{Acknowledgements}

I would like to thank the organizers of RADCOR 2009 for creating a pleasant and inspiring atmosphere. Special thanks goes to my colleagues from [51-53] for a fruitful collaboration. The work of the author was supported by SFB/TR 9 and by the Helmholtz-Alliance "Physics at the Terascale". 


\section{References}

[1] L. Magnea and G. Sterman, Phys. Rev. D 42 (1990) 4222.

[2] S. Catani, Phys. Lett. B 427 (1998) 161 [hep-ph/9802439].

[3] G. Sterman and M.E. Tejeda-Yeomans, Phys. Lett. B 552 (2003) 48 [hep-ph/0210130].

[4] L. J. Dixon, L. Magnea and G. Sterman, JHEP 0808, 022 (2008) [arXiv:0805.3515 [hep-ph]].

[5] L. Magnea, Nucl. Phys. B 593 (2001) 269 [hep-ph/0006255].

[6] S. Moch, J.A.M. Vermaseren and A. Vogt, JHEP 0508 (2005) 049 [hep-ph/0507039].

[7] T. Becher and M. Neubert, Phys. Rev. Lett. 102 (2009) 162001 [arXiv:0901.0722 [hep-ph]].

[8] E. Gardi and L. Magnea, JHEP 0903 (2009) 079 [arXiv:0901.1091 [hep-ph]].

[9] L. J. Dixon, Phys. Rev. D 79 (2009) 091501 [arXiv:0901.3414 [hep-ph]].

[10] C. Anastasiou, Z. Bern, L. J. Dixon and D. A. Kosower, Phys. Rev. Lett. 91, 251602 (2003) [arXiv:hep-th/0309040].

[11] Z. Bern, L. J. Dixon and V. A. Smirnov, Phys. Rev. D 72 (2005) 085001 [hep-th/0505205].

[12] Z. Bern, M. Czakon, D. A. Kosower, R. Roiban and V. A. Smirnov, Phys. Rev. Lett. 97 (2006) 181601 [hep-th/0604074].

[13] Z. Bern, L. J. Dixon, D. C. Dunbar, M. Perelstein and J. S. Rozowsky, Nucl. Phys. B 530, 401 (1998) [hep-th/9802162].

[14] Z. Bern, L. J. Dixon, M. Perelstein and J. S. Rozowsky, Nucl. Phys. B 546, 423 (1999) [hep-th/9811140].

[15] Z. Bern, L. J. Dixon and R. Roiban, Phys. Lett. B 644, 265 (2007) [hep-th/0611086].

[16] G. Kramer and B. Lampe, Z. Phys. C 34 (1987) 497; 42 (1989) 504(E).

[17] T. Matsuura and W.L. van Neerven, Z. Phys. C 38 (1988) 623.

[18] T. Matsuura, S.C. van der Maarck and W.L. van Neerven, Nucl. Phys. B 319 (1989) 570.

[19] R.V. Harlander, Phys. Lett. B 492 (2000) 74 [hep-ph/0007289].

[20] V. Ravindran, J. Smith and W.L. van Neerven, Nucl. Phys. B 704 (2005) 332 [hep-ph/0408315].

[21] T. Gehrmann, T. Huber and D. Maître, Phys. Lett. B 622 (2005) 295 [hep-ph/0507061].

[22] S. Moch, J.A.M. Vermaseren and A. Vogt, Phys. Lett. B 625 (2005) 245 [hep-ph/0508055].

[23] P. A. Baikov, K. G. Chetyrkin, A. V. Smirnov, V. A. Smirnov and M. Steinhauser, Phys. Rev. Lett. 102 (2009) 212002 [arXiv:0902.3519 [hep-ph]].

[24] B. Toedtli, arXiv:0903.0540 [hep-ph].

[25] F.V. Tkachov, Phys. Lett. 100B (1981) 65.

[26] K.G. Chetyrkin and F.V. Tkachov, Nucl. Phys. B192 (1981) 159.

[27] S. Laporta, Int. J. Mod. Phys. A 15 (2000) 5087 [hep-ph/0102033].

[28] C. Anastasiou and A. Lazopoulos, JHEP 0407 (2004) 046 [hep-ph/0404258].

[29] T. Gehrmann and E. Remiddi, Nucl. Phys. B 580 (2000) 485 [hep-ph/9912329]. 
[30] A. V. Smirnov, JHEP 0810 (2008) 107 [arXiv:0807.3243 [hep-ph]].

[31] M. Steinhauser, these proceedings

[32] S. Bekavac, Comput. Phys. Commun. 175 (2006) 180 [arXiv:hep-ph/0505174].

[33] S.G. Gorishnii, S.A. Larin, L.R. Surguladze and F.V. Tkachov, Comput. Phys. Comm. 55 (1989) 381; S.A. Larin, F.V. Tkachov and J.A.M. Vermaseren, NIKHEF-H-91-18.

[34] P.A. Baikov, Phys. Lett. B 634 (2006) 325 [hep-ph/0507053].

[35] T. Huber and D. Maître, Comput. Phys. Commun. 175 (2006) 122 [hep-ph/0507094].

[36] T. Huber and D. Maître, Comput. Phys. Commun. 178 (2008) 755 [arXiv:0708.2443 [hep-ph]].

[37] V.A. Smirnov, Phys. Lett. B 460 (1999) 397 [hep-ph/9905323].

[38] J.B. Tausk, Phys. Lett. B 469 (1999) 225 [hep-ph/9909506].

[39] V. A. Smirnov, "Evaluating Feynman integrals", Springer Tracts Mod. Phys. 211 (2004) 1.

[40] C. Anastasiou and A. Daleo, JHEP 0610 (2006) 031 [hep-ph/0511176].

[41] J. Gluza, K. Kajda and T. Riemann, Comput. Phys. Commun. 177 (2007) 879 [arXiv:0704.2423 [hep-ph]].

[42] M. Czakon, Comput. Phys. Commun. 175 (2006) 559 [hep-ph/0511200].

[43] A. V. Smirnov and V. A. Smirnov, Eur. Phys. J. C 62 (2009) 445 [arXiv:0901.0386 [hep-ph]].

[44] T. Binoth and G. Heinrich, Nucl. Phys. B 585 (2000) 741 [hep-ph/0004013].

[45] T. Binoth and G. Heinrich, Nucl. Phys. B 680 (2004) 375 [hep-ph/0305234].

[46] A. V. Smirnov and M. N. Tentyukov, Comput. Phys. Commun. 180 (2009) 735 [arXiv:0807.4129 [hep-ph]].

[47] D. Maître, Comput. Phys. Commun. 174 (2006) 222 [hep-ph/0507152]; D. Maître, hep-ph/0703052.

[48] D. A. Kosower, unpublished. An earlier version is available at http://projects.hepforge.org/mbtools/.

[49] J. A. M. Vermaseren, Int. J. Mod. Phys. A 14 (1999) 2037 [hep-ph/9806280].

[50] S. Moch, P. Uwer and S. Weinzierl, J. Math. Phys. 43 (2002) 3363 [hep-ph/0110083].

[51] T. Gehrmann, G. Heinrich, T. Huber and C. Studerus, Phys. Lett. B 640, 252 (2006) [hep-ph/0607185].

[52] G. Heinrich, T. Huber and D. Maître, Phys. Lett. B 662 (2008) 344 [arXiv:0711.3590 [hep-ph]].

[53] G. Heinrich, T. Huber, D. A. Kosower and V. A. Smirnov, Phys. Lett. B 678 (2009) 359 [arXiv:0902.3512 [hep-ph]].

[54] H.R.P. Ferguson, D.H. Bailey and S. Arno, Math. Comput. 68, (1999) 351, NASA-Ames Technical Report, NAS-96-005. 